\title{
Rapidity Dependence of HBT Radii Based on a Hydrodynamical Model
}

\author{
Kenji Morita ${ }^{1 *}$ \\ ${ }^{1}$ Department of Physics, Waseda University, Tokyo 169-8555, Japan
}

Received on 10 November, 2006

\begin{abstract}
We calculate two-pion correlation functions at finite rapidities based on a hydrodynamical model which does not assume explicit boost invariance along the collision axis. Extracting the HBT radii through $\chi^{2}$ fits in both Cartesian and Yano-Koonin-Podgoretskii parametrizations, we compare them with experimental results from the PHOBOS collaboration. Based on the results, we discuss longitudinal expansion dynamics.
\end{abstract}

Keywords: Hydrodynamical model; Pion interferometry; Boost invariance

\section{INTRODUCTION}

"Perfect fluidity" of the matter created at the Relativistic Heavy Ion Collider (RHIC) at BNL is some of the most exciting news in the field of high energy nuclear physics [1]. Experimental results and their comparison with theoretical calculation reveal that the matter created in $\mathrm{Au}+\mathrm{Au}$ collisions should be something like a liquid of quarks and gluons, unlike a gas of almost free partons as naively expected [2]. One strong piece of evidence for this finding is the observation of large elliptic flow $\left(v_{2}\right)$ and its agreement with a perfect fluiddynamical calculation [3]. In order to reproduce the experimental result with such models, an equation of state assuming a partonic state at high temperature and a phase transition and rapid thermalization time $\left(\tau_{0} \leq 1 \mathrm{fm} / c\right)$ are required [3]. The hydrodynamic model based on numerical solutions of the relativistic hydrodynamic equation for perfect fluid has become an indispensable tool for theoretical analyses of relativistic heavy ion collisions. Furthermore, the model itself has been becoming more sophisticated in order to reproduce new experimental data with higher statistics. Currently, the most sophisticated calculations model a full three-dimensional (solving hydrodynamic equation without any symmetry) hydrodynamic expansion followed by a hadronic cascade $[4,5]$. These models can reproduce most soft hadronic observables. Especially, the simultaneous description of particle ratios, transverse momentum spectra and elliptic flow is possible with such hybrid models.

However, there are still some insufficient ingredients in the hydrodynamic analyses. First, we don't have reasonable initial condition derived from first principles. Recently, the Color Glass Condensate (CGC) has been proposed as a suitable initial condition for relativistic heavy ion collisions [6]. This picture has been examined as an initial condition for a hydrodynamic model in Ref. [7] and found to give a good description of some observables in the case of fully hydrodynamic description of the collision process. However, this initial condition fails if one takes hadronic dissipation into account [4]. This fact suggests there is still open space for a dissipative partonic phase, or improvement of the initial condition.

${ }^{*}$ Present Address: Institute of Physics and Applied Physics, Yonsei University, Seoul 120-749, Korea
Second, the equation of state (EoS) of QCD matter has not yet been fully understood. Since one of the most important merit of using a hydrodynamic model is that it can be directly related to the EoS, detailed information on the EoS for all region of temperature and baryonic chemical potential is indispensable. As for RHIC energies, the net baryon number observed at midrapidity is small enough to neglect it [8]. Nevertheless, the EoS at finite baryonic chemical potential may play an important role in the forward rapidity region and in heavy ion collisions at lower energies. Because of the well-known difficulty of lattice QCD at finite baryonic chemical potential [9], lattice QCD calculations have not yet provided the complete solution. For vanishing baryonic chemical potential, the lattice equation of state clearly shows a different behavior from the free parton gas [10], and a lattice-inspired EoS has been implemented in hydrodynamic calculations [11].

At last, in spite of the success in most soft observables, results of the two-pion momentum intensity correlation from such hydrodynamical models do not yet agree with experimental data. According to the symmetry of the wave function of two identical bosons, the two-particle correlation function can be related to sizes of the source from which particles are emitted. This fact is known as Hanbury Brown-Twiss (HBT) effect. Because it concerns source sizes, which depend on momentum of particle pairs due to collective flow, the pion correlation function is a diagnostic tool for the space-time evolution of the matter. Since the disagreement was first found with a $(2+1)$-dimensional model with boost invariance along the collision axis [12], many extensions such as an explicit longitudinal expansion [13, 14], incorporating chemical freeze-out [14], chiral model EoS [15], an opaque source [16], fluctuating initial conditions and continuous freeze-out [17], have been examined. The discrepancy has been reduced, but the situation is still unsatisfactory. There are various possibilities for further improvements.

So far discussion on the HBT radii at the RHIC has been limited to midrapidity because of the acceptances of the two experiments, STAR and PHENIX. PHOBOS also has measured the two-pion correlation function. By virtue of the wider acceptance of the PHOBOS detector, measurements at non-zero rapidity windows has been performed, and the data are now available in Ref. [18]. For analyses of such data in terms of the Cartesian parameterization $[19,20]$, it should be noted that there exists an additional HBT radius called "outlong cross term" [21] which vanishes at midrapidity due to 
the symmetry. This radius contains information on the correlation between freeze-out points on the transverse plane and those on the longitudinal direction. Hence, it is expected that this quantity is sensitive to longitudinal expansion dynamics beyond the boost-invariant approximation. Similar considerations also hold for the Yano-Koonin-Podgoretskir parametrization which has three radius parameters and one velocity parameter called YK velocity $[22,23]$. The PHOBOS data also provide rapidity dependence of the YKP radii and YK velocity [18], which may impose a restriction on the longitudinal expansion dynamics. Indeed, the initial matter distribution as an input for hydrodynamic calculations has not yet been fixed. This is indicated by Hirano in Ref. [24], in which two different initial energy density distributions result in reasonable agreement with experimental data of pseudorapidity distribution of charged hadrons measured in $130 \mathrm{~A} \mathrm{GeV} \mathrm{Au+Au} \mathrm{collisions} \mathrm{at}$ RHIC.

In this work, we employ two different initial energy density distributions for the hydrodynamic equations, as in Ref. [24]. We focus our discussion on central collisions. Both of them are tuned so that they reproduce the pseudorapidity distribution of charged hadrons measured in the most central events at $200 \mathrm{~A} \mathrm{GeV} \mathrm{Au+Au} \mathrm{collisions.} \mathrm{Then,} \mathrm{we} \mathrm{compare} \mathrm{the} \mathrm{space-}$ time evolution and shape of the freeze-out hypersurface of the fluids and see how the difference in the initial condition is reflected onto them. We calculate the two-pion correlation function as the most promising experimental observable to see the difference. Extracting the HBT radii through Gaussian fits, we compare them with the experimental results and discuss the transverse momentum and rapidity dependence of the HBT radii. In the next section, we briefly review the hydrodynamical model used in this work. Initial conditions are given in Sec.III. In Sec. IV, we show numerical solutions of hydrodynamical equations for the initial conditions given in Sec. III. Results for the HBT radii as compared with the experimental data are given in Sec. V. Section VI is devoted to a summary.

\section{HYDRODYNAMICAL MODEL}

The basic equation of hydrodynamical models is the energy-momentum conservation law

$$
\partial_{\mu} T^{\mu v}=0
$$

where $T^{\mu v}$ is the energy-momentum tensor. For a perfect fluid,

$$
T^{\mu v}=(\varepsilon+P) u^{\mu} u^{v}-P g^{\mu v},
$$

where $g^{\mu \nu}=\operatorname{diag}(+,-,-,-)$ and $\varepsilon, P$ and $u^{\mu}$ are the energy density, pressure and the four velocities of the fluid, respectively. If one takes a conserved charge $i$ such as baryon number and strangeness into account, the conservation law

$$
\partial_{\mu}\left(n_{i} u^{\mu}\right)=0
$$

is added. Providing an $\operatorname{EoS} P=P\left(\varepsilon, n_{i}\right)$, one can solve these coupled equations numerically.

In this work, we consider the baryon number charge as a conserved charge and adopt an equation of state which exhibits a first order phase transition on the phase boundary in the $T-\mu_{\mathrm{B}}$ plane from the free massless partonic gas with three flavors to the free resonance gas which consists of hadrons except for hyperons up to $2 \mathrm{GeV} / c^{2}$ of mass with excluded volume correction[25]. See Ref.[26] for the detail. The critical temperature $T_{\mathrm{c}}$ at vanishing baryonic chemical potential is set to $160 \mathrm{MeV}$. This model is basically same as the one used in Refs. [13, 16].

Defining the $z$-axis as the collision axis, we use a cylindrical coordinate system $\left(\tau, \eta_{\mathrm{s}}, r, \phi\right)$ as follows;

$$
\begin{aligned}
t & =\tau \cosh \eta_{\mathrm{s}}, \\
z & =\tau \sinh \eta_{\mathrm{s}}, \\
r_{x} & =r \cos \phi, \\
r_{y} & =r \sin \phi .
\end{aligned}
$$

Here, $\tau=\sqrt{t^{2}-z^{2}}$ is the proper time and $\eta_{\mathrm{s}}=1 / 2 \ln [(t+$ $z) /(t-z)]$ is the space-time rapidity. Since we focus on central collisions, we assume an azimuthally symmetric system. Then, by virtue of $u_{\mu} u^{\mu}=1$, the four velocities are given in terms of a longitudinal flow rapidity $Y_{\mathrm{L}}$ and a transverse flow rapidity $Y_{\mathrm{T}}$ as

$$
\begin{aligned}
u^{\tau} & =\cosh \left(Y_{\mathrm{L}}-\eta_{\mathrm{s}}\right) \cosh Y_{\mathrm{T}}, \\
u^{\eta_{\mathrm{s}}} & =\sinh \left(Y_{\mathrm{L}}-\eta_{\mathrm{s}}\right) \cosh Y_{\mathrm{T}}, \\
u^{r} & =\sinh Y_{\mathrm{L}} .
\end{aligned}
$$

To solve the equations numerically, we employed a method based on the Lagrangian hydrodynamics which traces flux of the current. The numerical procedure is described in Ref. [27]. For treatment of the first order phase transition, we introduce a fraction of the volume of the QGP phase to express the energy density and net baryon number density at the mixed phase [26]. In this algorithm, we explicitly solve the entropy and baryon number conservation law. We checked that these quantities are conserved throughout the numerical calculation within $1 \%$ accuracy for a time step $\delta \tau=0.01 \mathrm{fm} / c$, by choosing proper mesh sizes of $\eta_{\mathrm{s}}$ and $r$ directions.

\section{INITIAL CONDITIONS}

Firstly, we choose an initial proper time as $\tau_{0}=1 \mathrm{fm} / c$. Initial values for other variables are given on this hyperbola. Longitudinal flow rapidity is set to the Bjorken's scaling ansatz $Y_{\mathrm{L}}=\eta_{\mathrm{s}}$ [28]. Transverse flow is simply neglected at the initial proper time [29]. For the matter distributions, we assume that the energy and baryon number density are proportional to the number of binary collisions. Hence, for the Woods-Saxon profile of the nucleon density in nuclei,

$$
\rho_{\mathrm{W}}(r, z)=\frac{\rho_{0}}{e^{\left(\sqrt{r^{2}+z^{2}}-R\right) / \xi}+1},
$$

where $R=1.12 A^{1 / 3}-0.86 A^{-1 / 3} \mathrm{fm}$ is the radius of the nuclear with mass number $A, \xi=0.54 \mathrm{fm}$ is the surface diffuseness and $\rho_{0}$ is the normal nuclear matter density, the density of binary collisions at vanishing impact parameter is given by

$$
n_{\mathrm{BC}}(r)=\sigma_{0}\left[\int_{-\infty}^{\infty} d z \rho_{\mathrm{W}}(r, z)\right]^{2},
$$


with $\sigma_{0}$ being the total inelastic nucleon-nucleon cross section which is absorbed into the proportionality constant between $n_{\mathrm{BC}}$ and matter distributions.

Then, the energy density distribution is parameterized with a "flat+Gaussian" form,

$$
\varepsilon\left(\tau_{0}, \eta_{\mathrm{s}}, r\right)=\varepsilon_{0} \exp \left[-\frac{\left(\left|\eta_{\mathrm{s}}\right|-\eta_{\mathrm{s} 0}\right)^{2}}{2 \sigma_{\eta_{\mathrm{s}}}^{2}} \theta\left(\left|\eta_{\mathrm{s}}\right|-\eta_{\mathrm{s} 0}\right)\right] \overline{n_{\mathrm{BC}}}(r) .
$$

Here, $\overline{n_{\mathrm{BC}}}(r)$ is the normalized density of binary collisions (12), $\varepsilon_{0}$ the maximum energy density, and $\eta_{\mathrm{s} 0}$ and $\sigma_{\eta_{\mathrm{s}}}$ are parameters which determine the length of the flat region and width of the Gaussian part, respectively. Similarly, the net baryon number density distribution is parameterized as

$$
\begin{aligned}
& n_{\mathrm{B}}\left(\tau_{0}, \eta_{\mathrm{s}}, r\right)=n_{\mathrm{B} 0}\left\{\exp \left[-\frac{\left(\left|\eta_{\mathrm{s}}\right|-\eta_{\mathrm{sD}}\right)^{2}}{2 \sigma_{\mathrm{sD}}^{2}}\right] \theta\left(\left|\eta_{\mathrm{s}}\right|-\eta_{\mathrm{s} 0}\right)\right. \\
& \left.\quad+\exp \left[-\frac{\left(\eta_{\mathrm{s} 0}-\eta_{\mathrm{sD}}\right)^{2}}{2 \sigma_{\mathrm{sD}}^{2}}\right] \theta\left(\eta_{\mathrm{s} 0}-\left|\eta_{\mathrm{s}}\right|\right)\right\} \overline{n_{\mathrm{BC}}}(r)
\end{aligned}
$$

where $n_{\mathrm{B} 0}$ is the maximum net baryon number density and $\eta_{\mathrm{sD}}$ and $\sigma_{\mathrm{sD}}$ are the shape parameters as in Eq. (13).

To calculate final particle distribution, we use the CooperFrye prescription [30]. The pseudorapidity distribution for a particle species $i$ is given by

$$
\frac{d N_{i}}{d \eta}=\frac{d_{i}}{(2 \pi)^{2}} \int_{0}^{\infty} d k_{t} \frac{k_{t}|\mathbf{k}|}{k^{0}} \int_{\Sigma} k \cdot d \sigma f\left(k \cdot u, T, \mu_{\mathrm{B}}\right),
$$

where $k^{\mu}$ is the momentum of thermally produced particles $i$ with $d_{i}$ being the number of degrees of freedom, the pseudorapidity $\eta$ defined by $\eta=1 / 2 \ln \left[\left(|\mathbf{k}|+k_{z}\right) /\left(|\mathbf{k}|-k_{z}\right)\right]$, and $f\left(k \cdot u, T, \mu_{\mathrm{B}}\right)$ describing the the equilibrium distribution functions. We take into account not only directly produced particles but also resonance decay contributions. The freezeout hypersurface $\Sigma$ is defined by picking a constant temperature, $T=T_{\mathrm{f}}=140 \mathrm{MeV}$. Here, we assume that thermal and chemical freeze-out occur simultaneously. Since experimental data of particle yields can be well described by the statistical model with high chemical freeze-out temperature close to $T_{\mathrm{c}}$ [31], we cannot reproduce the correct particle yields with this lower freeze-out temperature. However, in hydrodynamic analyses, $k_{t}$ spectra are sensitive to the thermal freeze-out temperature, which affects the transverse expansion. In this calculation, we set the freeze-out temperature so that pion $k_{t}$ spectrum is roughly reproduced in IC B and set the same freezeout temperature for IC A and IC B. Note that the freezeout temperature depends on the choice of the transverse profile of the initial matter distribution because a steeper pressure gradient yields larger transverse flow. For example, even $T_{\mathrm{f}} \simeq 150-160 \mathrm{MeV}$ is possible with an initialization based on pQCD+saturation model [32]. Our value is only slightly different from Ref. [24], where the initial profile is very similar. In order to reproduce both the particle yields and the $k_{t}$ spectra in dynamical regimes, one should introduce separate freeze-out temperatures $[14,33]$ or go to hybrid approach $[4,5,34,35]$. In this work, however, our main argument will

\begin{tabular}{|c|c|c|c|c|c|c|}
\hline & $\varepsilon_{0}\left[\mathrm{GeV} / \mathrm{fm}^{3}\right]$ & $\eta_{\mathrm{s} 0}$ & $\sigma_{\eta \mathrm{s}}$ & $n_{\mathrm{B} 0}\left[\mathrm{fm}^{-3}\right]$ & $\eta_{s \mathrm{D}}$ & $\sigma_{s \mathrm{D}}$ \\
\hline IC.A & 23.0 & 1.0 & 1.48 & 0.47 & 2.2 & 0.9 \\
\hline IC.B & 20.5 & 3.0 & 0.33 & 0.55 & 2.2 & 0.75 \\
\hline
\end{tabular}
not be so affected by the description of the freeze-out because we focus on longitudinal expansion.
TABLE I: Parameters in initial matter distributions.

In Table I, two sets of the initial parameters are listed. The corresponding initial energy density distributions, resultant pseudorapidity distributions and transverse momentum distributions are illustrated in Fig. 1, 2 and 3, respectively. We have chosen two initial conditions, both of which reproduce the experimental data of PHOBOS for the pseudo-rapidity distribution of charged hadrons [36], and of PHENIX for the transverse momentum distributions of $\pi^{-}, K^{-}$and $\bar{p}$ [37], and of BRAHMS for the rapidity distribution of net protons [38]. These two initial conditions are characterized by two parameters, $\eta_{\mathrm{s} 0}$ and $\sigma_{\mathrm{s} 0}$. One has small $\eta_{\mathrm{s} 0}$ and large $\sigma_{\mathrm{s} 0}$, which we denote initial condition A (IC.A). The other, which we represent IC.B, has the opposite feature; $\eta_{\mathrm{s} 0}$ is large and $\sigma_{\mathrm{s} 0}$ is small. The initial energy densities are both much larger than experimental estimations $\left(\sim 5 \mathrm{GeV} / \mathrm{fm}^{3}\right)$ based on Bjorken's formula [2], but note that $\varepsilon_{0}$ in Table I is not an average energy density but maximum energy density, which strongly depends on the profile of initial matter distributions [39]. We calculated pseudorapidity distributions for not only these two initial conditions, but also for intermediate ones by varying $\eta_{\mathrm{s} 0}$ from 1.0 to 3.0, and found that they can also reproduce the experimental data by adjusting other parameters appropriately. Perhaps the best fit will exist in the middle of this parameter range [40]. Here, we choose the extreme cases in order to see differences in the space-time evolution of the fluids originating from the difference in the initial conditions.

\section{SPACE-TIME EVOLUTION OF THE FLUIDS}

Figures 4 and 5 show the space-time evolution of the temperature distributions and deviation from the scaling solution $Y_{\mathrm{L}}=\eta_{\mathrm{s}}$, as a function of $\eta_{\mathrm{s}}$ at $r=0$ for various $\tau$, respectively. From these figures, we find that the space-time evolution at forward rapidity is quite different between IC.A and IC.B in spite of the fact that both solutions give similar pseudorapidity distributions of hadrons. In IC.B, the sharp decrease of temperature, which is identical to steep pressure gradient at forward rapidity, causes rapid acceleration of the longitudinal flow at the edge of the fluid. On the other hand, in IC.A, pressure gradient is rather gradual. Hence, the resultant deviation from the scaling solution is smaller. However, because pressure gradients exist at smaller $\eta_{\mathrm{s}}$ in IC.A, such deviations take place at $\eta_{\mathrm{s}} \simeq 1$ while the flow maintains the scaling solutions up to $\eta_{\mathrm{s}} \simeq 2$ in IC.B. This fact explains the slightly larger $\varepsilon_{0}$ in IC.A since faster longitudinal expansion than the scaling expansion pushes entropy per unit rapidity to forward rapidity $[13,41]$.

Although Figs. 4 and 5 show that there exist differences between IC.A and IC.B in the space-time evolution, it is not triv- 

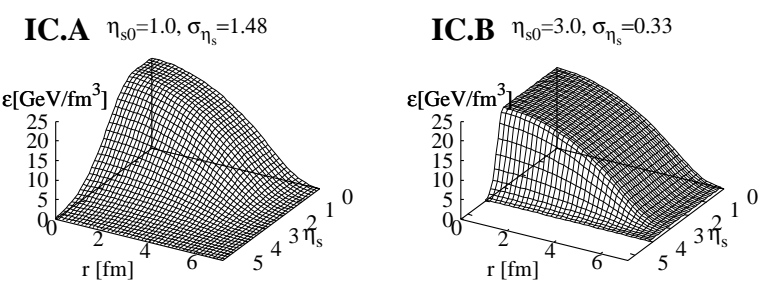

FIG. 1: Initial energy density distributions

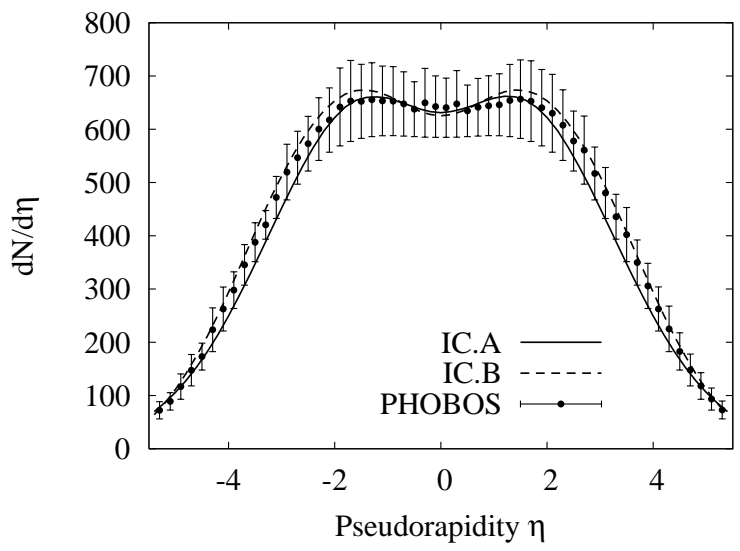

FIG. 2: Pseudorapidity distribution. The solid line and dashed line stand for the initial conditions A and B, respectively. Experimental data measured by PHOBOS are taken from Ref.[36].

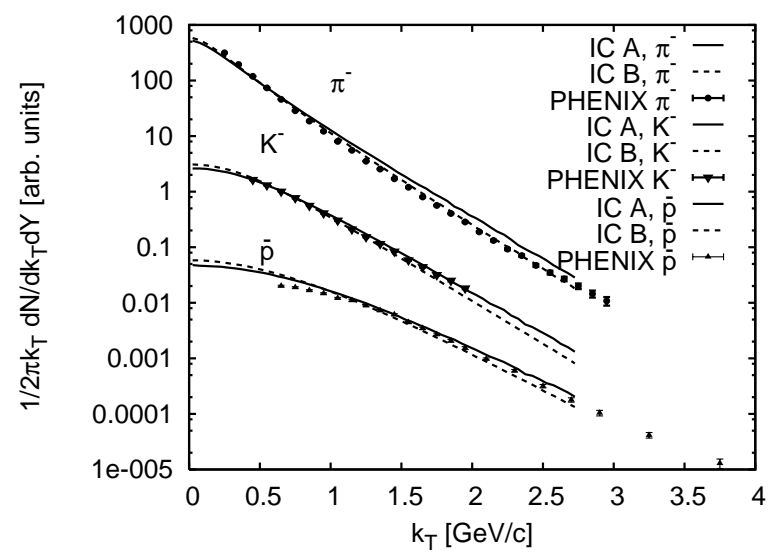

FIG. 3: Transverse momentum distribution of identified negatively charged hadrons. Spectra for kaons and anti-protons are scaled by factors 0.1 and 0.01 for a clear comparison of the slopes, respectively. Experimental data measured by PHENIX are taken from Ref. [37]. Identification of symbols is same as Fig. 2.

ial that such differences can survive at the freeze-out hypersurfaces. Since hadrons strongly interact and provide information only at thermal freeze-out, differences in the freeze-out hypersurfaces are necessary to lead to a signature in hadronic experimental observables.

We show the freeze-out proper time $\tau_{\mathrm{f}}$ of all fluid elements in Fig. 6. This characterizes the shape of the freeze-out hypersurface, which is expected to affect the HBT radii. In Fig. 6,
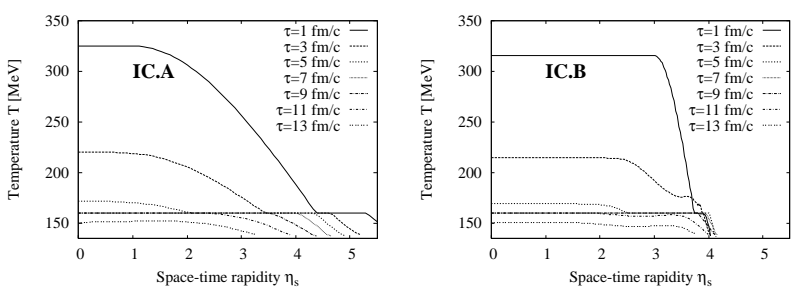

FIG. 4: Space-time evolution of temperature distributions at $r=0$.
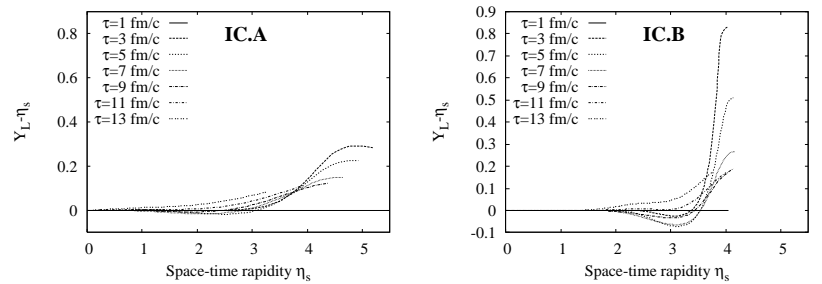

FIG. 5: Deviation from the scaling solution at $r=0$.
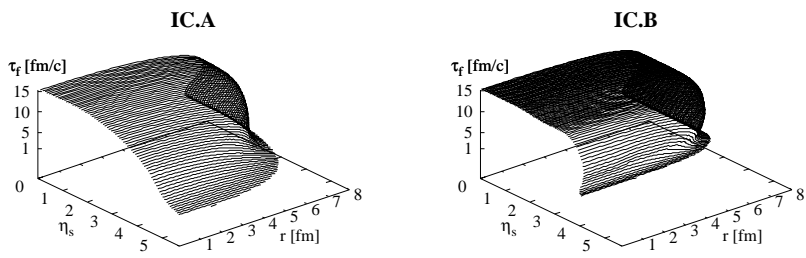

FIG. 6: Freeze-out hypersurface of the fluids.
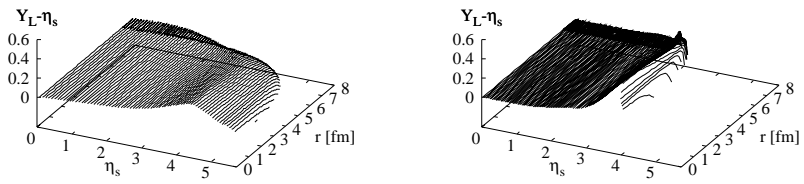

FIG. 7: Deviation from the scaling solution on the freeze-out hypersurfaces.

we can see that the system expands in the transverse direction in both of the fluids. Due to the same transverse profile, there is no apparent difference in the transverse direction. On the other hand, the shape of the hypersurface in the $\eta_{\mathrm{s}}$ direction shows some variations. In IC.B, expansion appears and the freeze-out proper time is mostly constant in the broad range of $\eta_{\mathrm{s}}$, while it moderately decreases with $\eta_{\mathrm{s}}$ in IC.A. This is a consequence of the different longitudinal flow profile (Fig. 5). We also plot the deviation from the scaling solution at the freeze-out in Fig. 7. The large deviation seen at forward rapidity in IC.B (Fig. 5) survives until freeze-out. We will see 
how these differences affect the HBT radii in the next section.

\section{HBT RADII}

\section{A. Two-pion correlation function}

Assuming that the source is completely chaotic, we can calculate the two-particle correlation momentum intensity correlation function through this formula [42]

$$
C_{2}(q, K)=1+\frac{|I(q, K)|^{2}}{I\left(0, k_{1}\right) I\left(0, k_{2}\right)},
$$

where $q=k_{1}-k_{2}$ is the four-relative momentum and $K=$ $1 / 2\left(k_{1}+k_{2}\right)$ is the four-average momentum, with $k_{i}$ being on-shell momentum of emitted pions. The interference term $I(q, K)$ can be chosen as

$$
I(q, K)=\int_{\Sigma} K \cdot d \sigma e^{i q \cdot x} f(u \cdot K, T),
$$

so that $I\left(0, k_{i}\right)$ reduces to the Cooper-Frye formula [43].

Experimentally, the two-pion correlation function is defined as

$$
C_{2}(\mathbf{q})=\frac{A(\mathbf{q})}{B(\mathbf{q})}
$$

where $A(\mathbf{q})$ is the measured two-pion pair distribution with momentum difference $\mathbf{q}$, and $B(\mathbf{q})$ is the background pair distribution generated from mixed events. Momentum acceptances are imposed separately in the numerator and the denominator. Accounting for the large acceptance in the PHOBOS experiment, $0.4<Y_{\pi \pi}<1.3$ for three $K_{\mathrm{T}}$ bins and $0.1<K_{\mathrm{T}}<1.4 \mathrm{GeV} / c$ for three rapidity bins, we integrate the correlation function as follows:

$$
\begin{gathered}
C\left(q ; K_{\mathrm{T}}\right)=1+\frac{\int_{0.4}^{1.3} d Y_{\pi \pi}|I(q, K)|^{2}}{\int_{0.4}^{1.3} d Y_{\pi \pi} I\left(0, k_{1}\right) I\left(0, k_{2}\right)}, \\
C\left(q ; Y_{\pi \pi}\right)=1+\frac{\int_{0.1}^{1.4} d K_{\mathrm{T}} K_{\mathrm{T}}|I(q, K)|^{2}}{\int_{0.1}^{1.4} d K_{\mathrm{T}} K_{\mathrm{T}} I\left(0, k_{1}\right) I\left(0, k_{2}\right)} .
\end{gathered}
$$

For simplicity, we consider only directly emitted pions and neglect resonance decay contributions.

\section{B. $K_{\mathrm{T}}$ dependence of the $\mathrm{HBT}$ radii in the Cartesian parametrization}

Physical meaning of the HBT radii depends on the choice of three independent components of the relative momentum $q$. The most standard choice is the so-called Cartesian BertchPratt parametrization $[19,20] \mathbf{q}=\left(q_{\text {out }}, q_{\text {side }}, q_{\text {long }}\right)$ in which "long" means parallel to the collision axis, "side" perpendicular to the transverse component of the average momentum $\mathbf{K}_{\mathrm{T}}$ and "out" parallel to $\mathbf{K}_{\mathrm{T}}$. In the case of azimuthally symmetric system as considered here, one can put $\mathbf{K}_{\mathrm{T}}=\left(K_{\mathrm{T}}, 0\right)$ so that $q_{\text {out }}=q_{x}$ and $q_{\text {side }}=q_{y}$. Note that $q_{\text {long }}=q_{z}$. Then, the
Gaussian form of the two-pion correlation function is given as [21]

$$
\begin{gathered}
C_{2 \text { fit }}(\mathbf{q})=1+\lambda \exp \left(-q_{\text {out }}^{2} R_{\text {out }}^{2}-q_{\text {side }}^{2} R_{\text {side }}^{2}-q_{\text {long }}^{2} R_{\text {long }}^{2}\right. \\
\left.-2 q_{\text {out }} q_{\text {long }} R_{\text {ol }}^{2}\right) .
\end{gathered}
$$

The HBT radii $R_{i}$ can be extracted by a $\chi^{2}$-fit to the above fitting function. For a chaotic source, the chaoticity parameter $\lambda$ should become unity. However, the experimentally observed chaoticity is smaller than 1 because of such contributions as long-lived resonance decay [44]. Here we fix $\lambda=1$ in the Gaussian fit to the calculated correlation functions with Eqs. (19) and (20).

By expanding the correlation function (16) for $q \cdot x \ll 1$, the size parameters $R_{i}$ can be related to second order moments of the source function [21]. In the Cartesian parametrization, taking the longitudinal co-moving system (LCMS) makes the expression simple;

$$
\begin{aligned}
R_{\text {out }}^{2} & =\left\langle\left(\tilde{r_{x}}-\beta_{\perp} \tilde{t}\right)^{2}\right\rangle \\
& =\left\langle{\tilde{r_{x}}}^{2}\right\rangle-2 \beta_{\perp}\left\langle\tilde{r_{x}} \tilde{t}\right\rangle+\beta_{\perp}^{2}\left\langle\tilde{t}^{2}\right\rangle . \\
R_{\text {side }}^{2} & =\left\langle{\tilde{r_{y}}}^{2}\right\rangle, \\
R_{\text {long }}^{2} & =\left\langle\tilde{z}^{2}\right\rangle, \\
R_{\text {ol }}^{2} & =\left\langle\left(\tilde{r_{x}}-\beta_{\perp} \tilde{t}\right) \tilde{z}\right\rangle,
\end{aligned}
$$

where

$$
\langle A(x)\rangle \equiv \frac{\int_{\Sigma} k \cdot d \sigma f(u \cdot k, T) A(x)}{\int_{\Sigma} k \cdot d \sigma f(u \cdot k, T)},
$$

$\tilde{x} \equiv x-\langle x\rangle$, and $\beta_{\perp}=k_{\mathrm{T}} / E_{\mathbf{k}}$. Hence, $R_{\text {out }}, R_{\text {side }}$ and $R_{\text {long }}$ can be interpreted as a mixture of the thickness of the source and the emission duration, the transverse source size, and the longitudinal source size, as seen from the LCMS, respectively. The validity of these expressions for a hydrodynamical model is discussed in Ref. [45]. Although they have been shown to be good approximations, it is also pointed out that there are still some discrepancies, and one should use fitted HBT radii for comparison with the experimental data which are obtained from the fit [46].

Figure 8 shows results for the four HBT radii compared with the experimental data measured by PHOBOS [18]. For comparison of the initial conditions, any qualitative and quantitative difference cannot be seen in $R_{\text {out }}$ and $R_{\text {side }}$, as expected from Fig. 6 . $R_{\text {long }}$ of IC.A is about $1 \mathrm{fm}$ smaller than that of IC.B. This can be considered as a consequence of the fact that the deviation from the scaling solution at small $\eta_{\mathrm{s}}$ is larger in IC.A, because faster flow causes more thermal suppression of the emission region [13]. For these three radii, our calculation cannot reproduce the experimental results and show similar behavior with other perfect fluid dynamical calculations of Ref.[12-14, 16]. Especially $R_{\text {long }}$ shows the largest deviation from experimental data, although the calculation is improved by including longitudinal expansion without explicit boost invariance $[13,14]$. In the bottom of Fig. 8, the result of the out-long cross term is presented. Reflecting the uniform shape of the freeze-out hypersurface in Fig. 6, the value of $R_{\mathrm{ol}}^{2}$ 

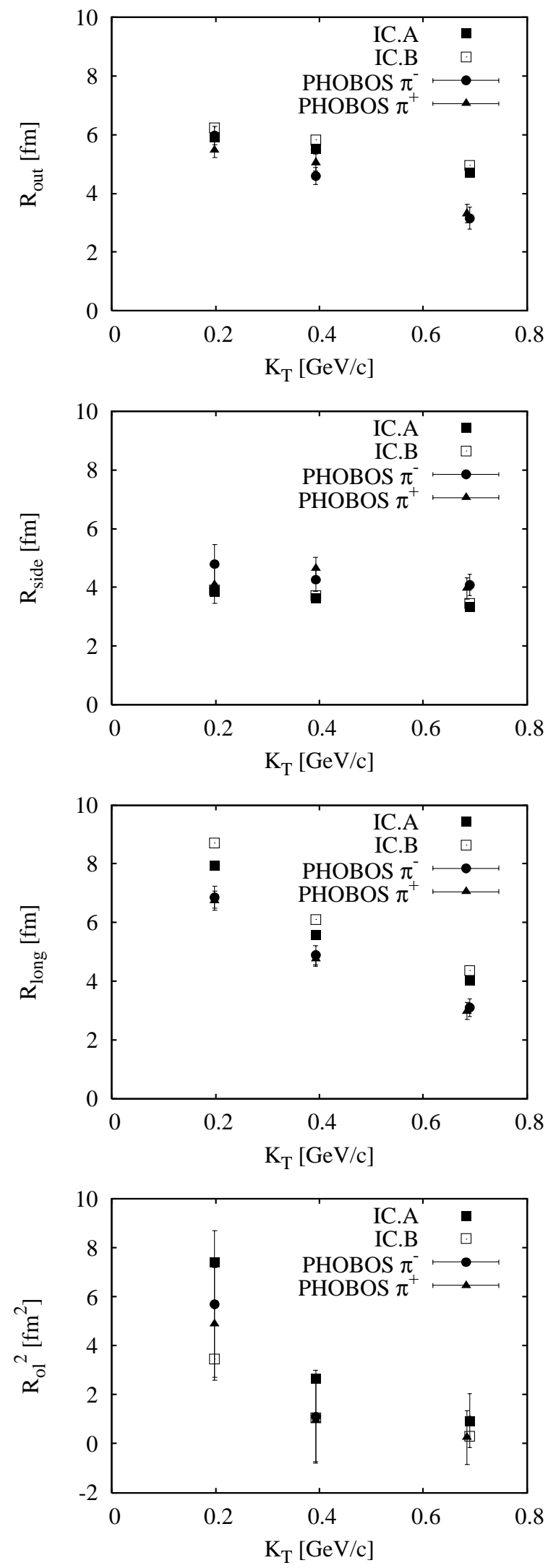

FIG. 8: $K_{\mathrm{T}}$ dependence of Cartesian HBT radii. Closed squares and open squares denote our results for IC.A and IC.B, respectively. Experimental data are taken from Ref. [18]. Error-bars for the experimental data are statistical only. of IC.B is smaller than that of IC.A. At the lowest $K_{\mathrm{T}}$ bin, the difference is about $4 \mathrm{fm}^{2}$. Unfortunately, experimental uncertainty is still too large to distinguish which initial condition is favored. However, it should be noted that both of two results agree with the experimental data, in spite of the disagreement of other radii.

\section{Rapidity dependence of the HBT radii in the YKP parametrization}

In the YKP parametrization, three independent components of the relative momentum $q$ are $q_{\perp}=\sqrt{q_{x}^{2}+q_{y}^{2}}, q_{\|}=q_{z}=$ $q_{\text {long }}$ and $q_{\tau}=E_{1}-E_{2}$. Then, the Gaussian fitting correlation function is given as

$$
\begin{aligned}
C_{2 \mathrm{YKP}}(\mathbf{q})= & 1+\lambda \exp \left[-R_{\perp}^{2} q_{\perp}^{2}-R_{\|}^{2}\left(q_{\|}^{2}-q_{\tau}^{2}\right)\right. \\
& \left.-\left(R_{\tau}^{2}+R_{\|}^{2}\right)(q \cdot U)^{2}\right],
\end{aligned}
$$

where $U^{\mu}=\gamma\left(1,0,0, v_{\mathrm{YK}}\right), \gamma=1 / \sqrt{1-v_{\mathrm{YK}}^{2}}$ and $v_{\mathrm{YK}}$ is the fourth fitting parameter called the YK velocity. The three HBT radii, $R_{\perp}, R_{\|}$and $R_{\tau}$ are invariant under a longitudinal boost. Physical meaning of the parameters can be given in a similar manner [23] and becomes the simplest as follows, if one adopt the $\mathrm{YK}$ frame where $v_{\mathrm{YK}}=0$,

$$
\begin{aligned}
R_{\perp}^{2} & =\left\langle\tilde{r}_{y}^{2}\right\rangle=R_{\text {side }}^{2}, \\
R_{\|}^{2} & \simeq\left\langle\tilde{z}^{2}\right\rangle=R_{\text {long }}^{2}, \\
R_{\tau}^{2} & \simeq\left\langle\tilde{t}^{2}\right\rangle .
\end{aligned}
$$

The main advantage of using YKP parametrization is that the three HBT radii directly give the transverse, longitudinal and temporal source size, that are seen from the YK frame. However, one should note that the latter two, (29) and (30), are approximate expressions which hold only if the source is not opaque [45]. Hence, $R_{\|}$and $R_{\tau}$ cannot be always regarded as the source sizes in the presence of strong transverse flow which makes the source highly opaque [16]. The general expression of $v_{\mathrm{YK}}$ is complicated one [23] but it can be regarded as a longitudinal flow velocity of the source measured in an observer's frame.

We plot results of HBT radii for the YKP parametrization in Fig. 9. Though PHOBOS measures only at small values of rapidity, we calculate the HBT radii for $Y_{\pi \pi}=$ $0.602,0.877,1.122,1.5,2.0,2.5,3.0,3.5$ and 4.0 and show the results as a prediction. For comparison between IC.A and IC.B, $R_{\perp}$ seems to barely reflect the uniform structure along $\eta_{\mathrm{s}}$ direction in IC.B. While $R_{\|}$shows a difference of order 1 fm at small rapidity coming from the deviation of the scaling solution as well as in the third panel of Fig. $8, R_{\tau}$ shows little difference and agrees with experiment. Large experimental errors will be due to the known difficulty of the limited kinematic region in the YKP parameterization [47]. Because of the large $K_{\mathrm{T}}$ window of the data, it is difficult to estimate the geometrical opacity effect on $R_{\tau}$. If we assume this effect is small, 



FIG. 9: HBT radii for the YKP parametrization. The identification of the symbols is the same as in Fig. 8.

a possible origin of the deviation of our result from the data is larger emission duration. Some model calculations based on source parametrization [48] and parametric exact solution of hydrodynamics [49] show very small emission duration time, $0-2 \mathrm{fm} / c$ in agreement with data on the bottom panel of Fig. 9. We cannot see any significant differences in the HBT radii at forward rapidity expected from Figs. 6 and 7 which display the differences of the source shape and the longitudinal flow. This will come from the fact that the number of produced particles is larger at late freeze-out proper time in the case of the current freeze-out condition [45].

Finally, the Yano-Koonin rapidity $Y_{\mathrm{YK}}=1 / 2 \ln [(1+$ $\left.\left.v_{\mathrm{YK}}\right) /\left(1-v_{\mathrm{YK}}\right)\right]$ is shown as a function of $Y_{\pi \pi}$ in Fig. 10. Both results from IC.A and IC.B surprisingly agree with the exper- imental data and show no difference between the two. In the forward rapidity region, our results show deviation from the infinite boost invariant case, which is indicated by the straight line. Although our solutions of longitudinal flow show deviation from the scaling solution (Figs. 5 and 7), the result would have to yield $Y_{\mathrm{YK}}$ larger than a given $Y_{\pi \pi}$, if $Y_{\mathrm{YK}}$ correctly represents the longitudinal source velocity. Hence, this deviation will be caused by the finite size effect [45] which becomes more significant at forward rapidity rather than the difference in the flow velocity.

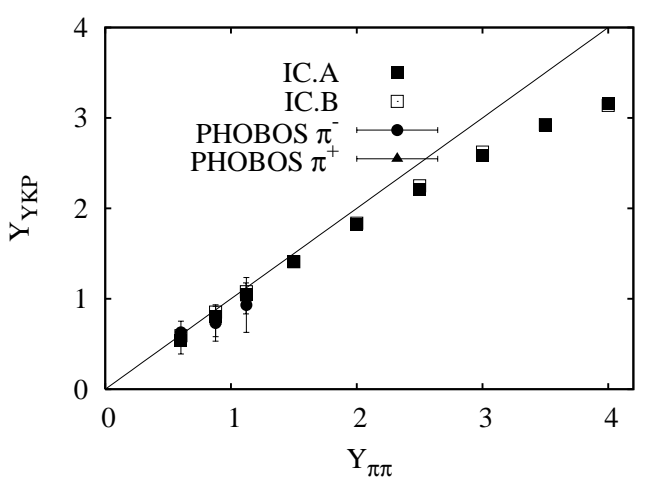

FIG. 10: The Yano-Koonin rapidity $Y_{\mathrm{YK}}$. The identification of the symbols is the same as in Figs. 8 and 9. The solid line indicates the case of the infinite boost-invariant source.

\section{SUMMARY}

In summary, we calculated the two-pion correlation function for two sources which are given by a hydrodynamical model without explicitly boost invariance along the collision axis. The two initial conditions are so chosen that both of them give consistent pseudorapidity distribution with the experimental data and have different shape in the longitudinal direction. Other model ingredient, initial transverse profile from the binary collision model, scaling solution for initial longitudinal flow, vanishing initial transverse flow, EoS with first order phase transition and Cooper-Frye freeze-out prescription with $T_{\mathrm{f}}=140 \mathrm{MeV}$ are the same in the two solutions. We find that there exist some differences in the space-time evolution of the fluids in spite of the fact that both fluids give similar particle distribution. The HBT radii are extracted from the two-pion correlation functions and compared with the experiment. In the Cartesian parametrization, the out-long cross term which arises at nonzero rapidities shows a difference between two initial conditions and the good agreements with the experimental data. The correlation function is also analyzed with the YKP parametrization. We find a small difference between the two initial conditions in $R_{\|}$which reflects deviation from the scaling solution in the longitudinal expansion as well as $R_{\text {long }}$ in the Cartesian parametrization. Possible sources of this disagreement are followings: EoS of current use exhibits first order phase transtion which makes the lifetime of the fluid longer, and assumes hadronic states is in fully chemical equilibrium. It is known that both crossover EoS [15] and incorporating chemical freeze-out [14] improve the lifetime 
then $R_{\text {long }}$ and $R_{\|}$. We used the conventional Cooper-Frye prescription for the freeze-out. Improvement of the freezeout prescription by continuous freeze-out [11] and hybrid approach $[4,5]$ can yield larger $R_{\text {side }}$ but this may lead to larger $R_{\text {out }}$ because of extended emission duration. Nevertheless, as a transport calculation [50] shows, positive $x-t$ correlation in the source function may resolve this problem. Finally, in spite of the disagreement of the HBT radii, the YK rapidity shows a good agreement with the experimental data. Our calculation predicts some deviations at larger rapidities from the infinite boost-invariant case. Hence, measurements at this region is needed for further understanding of the expansion dynamics.

\section{Acknowledgements}

The author is indebted to Profs. I. Ohba and H. Nakazato for their encourgement. He also would like to thank S. Muroya and T. Hirano for their helpful discussions. This work was supported by a Grant for the 21 st Century COE Program at Waseda University from Ministry of Education, Culture, Sports, Science and Technology of Japan. This is also supported by BK21 (Brain Korea 21) program of the Korean Ministry of Education.
[1] http://www.bnl.gov/bnlweb/pubaf/pr/PR_ _display.asp? prID $=05-38$

[2] I. Arsene et al., (BRAHMS Collaboration), Nucl. Phys. A 757, 1 (2005); B. B. Back et al., (PHOBOS Collaboration), ibid, A 757, 28 (2005); J. Adams et al., (STAR Collaboration), ibid, A 757, 102 (2005); K. Adcox et al., (PHENIX Collaboration), ibid, A 757, 184 (2005).

[3] P. F. Kolb, P. Huovinen, U. Heinz, and H. Heiselberg, Phys. Lett. B 500, 232 (2001).

[4] T. Hirano, U. Heinz, D. Kharzeev, R. Lacey, and Y. Nara, Phys. Lett. B 636, 299 (2006).

[5] C. Nonaka, S. A. Bass, arXiv:nucl-th/0607018.

[6] E. Iancu, R. Venugopalan, in Quark-Gluon Plasma 3, edited by R. C. Hwa, X. N. Wang, World Scientific, Singapore, 2004.

[7] T. Hirano and Y. Nara, Nucl. Phys. A 743, 305 (2004).

[8] I. G. Bearden et al., (BRAHMS Collaboration), Phys. Rev. Lett. 93, 102301 (2004).

[9] For a review, S. Muroya, A. Nakamura, C. Nonaka, and T. Takaishi, Prog. Theor. Phys. 110, 615 (2003).

[10] Y. Aoki, Z. Fodor, S. D. Katz, and K. K. Szabo, JHEP 0601, 089 (2006)

[11] Y. Hama, R. P. G. Andrade, F. Grassi, O. Socolowski Jr., T. Kodama, B. Tavares, and S. S. Padula, Nucl. Phys. A 774, 169 (2006).

[12] U. Heinz, P. Kolb, Nucl. Phys. A 702, 269 (2002).

[13] T. Hirano, K. Morita, S. Muroya, and C. Nonaka, Phys. Rev. C 65, 061902(R) (2002); K. Morita, S. Muroya, C. Nonaka, and T. Hirano, ibid., 66, 054904 (2002).

[14] T. Hirano, K. Tsuda, Phys. Rev. C 66, 054905 (2002).

[15] D. Zschiesche, H. Stöcker, W. Greiner, and S. Schramm, Phys. Rev. C 65, 064902 (2002).

[16] K. Morita, S. Muroya, Prog. Theor. Phys. 111, 93 (2004).

[17] O. Socorowski,Jr., F. Grassi, Y. Hama, and T. Kodama, Phys. Rev. Lett. 93, 182301 (2004).

[18] B. B. Back et al., (PHOBOS Collaboration), Phys. Rev. C 73, 031901(R) (2006).

[19] G. Bertsch, M. Gong, and M. Tohyama, Phys. Rev. C 37, 1896 (1988).

[20] S. Pratt, T. Csörgő, and J. Zimányi, Phys. Rev. C 42, 2646 (1990).

[21] S. Chapman, P. Scotto, and U. Heinz, Phys. Rev. Lett. 74, 4400 (1995).

[22] F. Yano, S. Koonin, Phys. Lett. B 78, 556 (1978); M. I. Podgoretskiı̌, Sov. J. Nucl. Phys. 37, 272 (1983).

[23] Y. -F. Wu, U. Heinz, B. Tomášik, and U. A. Wiedemann, Eur. Phys. J. C 1, 599 (1998).

[24] T. Hirano, Phys. Rev. C 65, 011901(R) (2001).

[25] Note that, however, this EoS does not agree with recent lattice
QCD calculations which exhibit cross-over transition at vanishing and small $\mu_{\mathrm{B}}[10]$.

[26] C. Nonaka, E. Honda, and S. Muroya, Eur. Phys. J. C 17, 663 (2000).

[27] T. Ishii, S. Muroya, Phys. Rev. D 46, 5156 (1992).

[28] J. D. Bjorken, Phys. Rev. D 27, 140 (1983).

[29] Note that the existence of the initial transverse flow can improve results for transverse momentum spectra but is an open issue. In this work, however, we simply neglect it in order to avoid to add additional parameters such as flow strength and profile. Since we are mainly focusing on longitudinal expansion dynamics in this paper, this simplification will not affect our main argument.

[30] F. Cooper and G. Frye, Phys. Rev. D 10, 186 (1974).

[31] P. Braun-Munzinger, D. Magestro, K. Redlich, and J. Stachel, Phys. Lett. B 518, 41 (2001).

[32] K. J. Eskola, H. Niemi, P. V. Ruuskanen, and S. S. Räsänen, Phys. Lett. B 566, 187 (2003).

[33] P. F. Kolb, R. Rapp, Phys. Rev. C 67, 044903 (2003).

[34] S. A. Bass, A. Dumitru, Phys. Rev. C 61, 064909 (2000).

[35] D. Teaney, J. Lauret, and E. V. Shuryak, Phys. Rev. Lett. 86, 4783 (2003).

[36] B. B. Back et al., (PHOBOS Collaboration), Phys. Rev. Lett. 91, 052303 (2003)

[37] S. S. Adler et al., (PHENIX Collaboration), Phys. Rev. C 69, 034909 (2004).

[38] I. G. Bearden et al., Phys. Rev. Lett. 93, 102301 (2004).

[39] P. F. Kolb, U. Heinz, P. Huovinen, K. J. Eskola, and K. Tuominen, Nucl. Phys. A 696, 197 (2001).

[40] L. M. Satarov, A. V. Merdeev, I. N. Mishustin, and H. Stöcker, arXiv:hep-ph/0606074

[41] K. J. Eskola, K. Kajantie, and P. V. Ruuskanen, Eur. Phys. J. C 1, 627 (1998).

[42] E. V. Shuryak, Phys. Lett. B 44, 387 (1973).

[43] S. Chapman, U. Heinz, Phys. Lett. B 340, 250 (1994).

[44] K. Morita, S. Muroya, and H. Nakamura, Prog. Theor. Phys. 114, 583 (2005); ibid, 116, 329 (2006). See also references therein.

[45] K. Morita, S. Muroya, H. Nakamura, and C. Nonaka, Phys. Rev. C. 61, 034904 (2000).

[46] E. Frodermann, U. Heinz, and M. A. Lisa, Phys. Rev. C 73, 044908 (2006).

[47] B. Tomášik and U. Heinz, Acta. Phys. Slov. 49, 251 (1999).

[48] F. Retiere, M. A. Lisa, Phys. Rev. C 70, 044907 (2004).

[49] M. Csanád, T. Csörgó, and B. Lörstad, Nucl. Phys. A 742, 80 (2004).

[50] Z. W. Lin, C. M. Ko, and S. Pal, Phys. Rev. Lett. 89, 152301 (2002). 